\title{
The Clinical Characteristics of Acute Myocardial Infarction with Ventricular Septal Perforation and the Prognosis Comparison of Different Treatment Methods
}

\author{
Chun Fu, MD, ${ }^{1}$ Qing Gao, MD, ${ }^{2}$ Zhou Zhao, $M D,{ }^{2}$ Yu Chen, $M D,{ }^{2}$ Jian Liu, MD, ${ }^{3}$ Youzhong An, $\mathrm{MD}^{1}$ \\ ${ }^{1}$ Critical Medicine Department, Peking University People's Hospital, Beijing, China; \\ ${ }^{2}$ Cardiac Surgery Department, Peking University People's Hospital, Beijing, China; \\ ${ }^{3}$ Cardiovascular Medicine Department, Peking University People's Hospital, Beijing, China
}

\section{ABSTRACT}

Objective: To explore the clinical characteristics of acute myocardial infarction (AMI) with ventricular septal perforation (VSR), the prognosis comparison of different treatment methods, and analysis of related risk factors.

Methods: From January 2006 to February 2020, 29 patients with AMI and VSR diagnosed in the People's Hospital of Peking University were selected as the study group. Among them, 16 cases were male $(55.2 \%), 13$ cases were female $(44.8 \%)$, and the average age was $64.69 \pm 10.32$ years old. They were divided into two groups: the survival group ( $N$ $=16)$ and non-survival group $(N=13)$, according to whether they survived within 30 days of surgical or drug conservative treatment. The clinical characteristics, coronary angiography, and treatment of the two groups were summarized, and the prognosis and related risk factors were analyzed.

Results: There was no significant difference in the basic clinical characteristics between the two groups $(P>0.05)$. Compared with the results of coronary angiography in the two groups, the proportion of the culprit vessel, which was a simple anterior descending branch in the non-survival group, was higher than that in the survival group. There was a statistical difference between the two groups $(P<0.05)$. The perioperative data of the two groups showed that the proportion of patients with complete revascularization, simultaneous bypass, and recanalization of culprit vessels in the survival group was significantly higher than that in the non-survival group $(P<0.05)$. However, the incidence of postoperative low cardiac output and mortality during hospitalization in the survival group were significantly lower than those in the non-survival group $(P<0.05)$. Logistic regression analysis showed that complete revascularization $(\mathrm{OR}=0.021,95 \% \mathrm{CI}$ $0.001-0.374, P=0.009)$ and recanalization of culprit vessels (OR $=0.045,95 \%$ CI $0.004-0.548, P=0.015)$ were independent risk factors for 30-day mortality. Kaplan-Meier survival curve showed that during the follow-up period, the long-term

Received April 26, 2021; accepted fune 21, 2021.

Correspondence: Zhou Zhao, MD, Cardiac Surgery Department, Peking University People’s Hospital, Beijing, China, 100044 (e-mail: chevo888@163.com). survival rate of patients with operation and complete revascularization was significantly higher than that of patients with drug conservative treatment and incomplete revascularization. There was a statistical difference between the two groups $(P<0.05)$.

Conclusion: Complete revascularization and recanalization of culprit vessels are independent risk factors for 30-day mortality in patients with AMI and VSR. The long-term survival rate of patients after surgery and complete revascularization is significantly higher than that of patients with conservative medical treatment and incomplete revascularization. Surgery and complete revascularization are important factors affecting the long-term prognosis of patients with AMI and VSR.

\section{INTRODUCTION}

VSR is a fatal complication after acute myocardial infarction (AMI). It's a great challenge to surgery and, at present, the incidence of VSR is reported to be between $0.17 \%$ and $0.31 \%$ [Crenshaw 2000]. Although the prognosis of AMI patients has greatly improved in recent years, the overall prognosis of VSR patients still is poor. The 30-day mortality rate of VSR patients treated with drug conservative therapy is still close to $100 \%$, and the 30 -day mortality rate of patients treated with surgery is close to $50 \%$ [Coskun 2009; Pang 2013]. This study conducted a retrospective analysis of 29 patients with AMI combined with VSR, aiming to explore and analyze the relevant risk factors affecting prognosis and guide clinical practice.

\section{DATA AND METHODS}

Research object: From January 2006 to February 2020, 29 patients with AMI and VSR diagnosed in the Heart Surgery Department of the People's Hospital of Peking University were selected as the subjects of study, including 16 males $(55.2 \%)$ and 13 females (44.8\%), with an average age of 64.69 \pm 10.32 years. Inclusion criteria: all patients had a clear history of severe chest pain; ST segment elevation $\geq 0.1 \mathrm{mv}$ (limb lead) or $0.2 \mathrm{mv}$ (chest lead); changes in at least two adjacent or related leads of ECG were performed in the external hospital or the People's Hospital of Peking University, and cardiac enzymes were increased; physical examination found that 
systolic murmurs could be heard in the left side of the sternum 3 to 4 intercostal space; echocardiography indicated that there was a clear left to right shunt in the ventricular level. Exclusion criteria: tumor patients or diseases that put patients in a cachexia state in a short period of time; diseases that progress rapidly with life expectancy $\leq 6$ months; combined with aortic wall hematoma or aortic dissection, aneurysm, arteriovenous malformation, etc.; combined with cardiomyopathy, such as hypertrophic cardiomyopathy, etc.

Research methods: Collected the general data (gender, age, etc.) of the patients. Laboratory indexes, including myocardial injury markers, triglyceride (TG), cholesterol (Chol), and low density lipoprotein (LDL), were collected in addition to echocardiographic results (such as the location and size of the perforation, end diastolic diameter of left ventricle, whether there was ventricular aneurysm, etc.), previous history (smoking history, hypertension history, old myocardial infarction history, diabetes history, etc.), coronary artery disease and perioperative situation, etc. Patients were divided into the survival group $(\mathrm{N}=$ 16) and non-survival group $(\mathrm{N}=13)$, according to whether they survived within 30 days after operation or drug conservative treatment. Summarized the clinical characteristics, coronary angiography and treatment of the two groups of patients and analyzed the prognosis and related risk factors.

Observation index: To record and compare the general data, hospital laboratory indexes, coronary angiography, treatment, 30-day mortality related risk factors, and longterm survival rate between the two groups.

Statistical methods: SPSS 22.0 statistical software was used to process the data. The measurement data of normal distribution is represented by mean \pm standard deviation ( $\mathrm{x}$ \pm s), and the comparison between groups is represented by $t$ test. The measurement data of non-normal distribution is represented by $\mathrm{M}(\mathrm{Q} 1, \mathrm{Q} 3)$, and the comparison between the two groups is represented by non-parameter test. The use case (percentage) of count data was expressed, and $\chi^{2}$ test was used for comparison between the two groups. The influencing factors of 30-day mortality were analyzed by binary regression and Kaplan Meier $(\mathrm{K}-\mathrm{M})$ method. $P<0.05$ was statistically significant.

\section{RESULTS}

Comparison of general data between the two groups: There was no significant difference between the two groups in gender, age, BMI, smoking, hypertension and diabetes, and previous myocardial infarction $(P>0.05)$. There was no significant difference in blood pressure, heart rate, cholesterol, triglyceride, low-density lipoprotein and high-density lipoprotein between the two groups $(P>0.05)$. (Table 1)Comparison of myocardial enzymology, echocardiography, and coronary angiography between the two groups: Comparing the myocardial enzyme indexes of the two groups, we found that there was statistical difference in creatine kinase (CKMB) immediately after admission $(P<0.05)$, but no significant difference in other indexes $(P>0.05)$.

There was no significant difference in VSR perforation location, perforation size, ejection fraction, and left ventricular end diastolic diameter between the two groups $(P>0.05)$.

Table 1. Patient characteristics

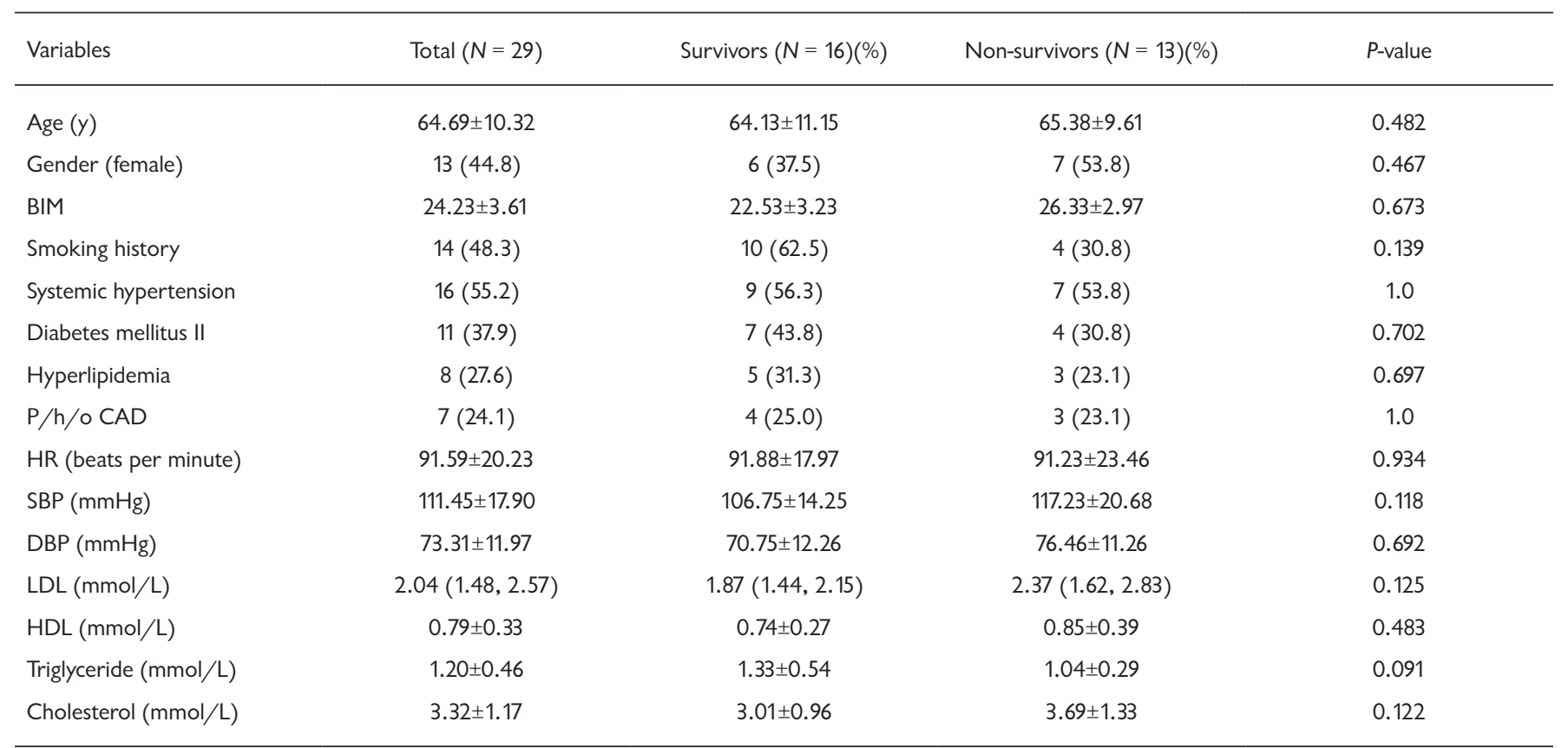

BMI, body mass index; CAD, coronary artery disease; P/h/o, past history of; HR, heart rate; SBP, systolic blood pressure; DBP, diastolic blood pressure; LDL, low density lipoprotein; HDL, high density lipoprotein. *Statistically significant 
Comparing the results of coronary angiography between the two groups, it was found that $75 \%(12 / 16)$ of the patients in the survival group had coronary angiography before operation, all of them were lesions of two or more coronary arteries; $61.5 \%(8 / 13)$ of the patients in the non-survival group had coronary angiography before operation, and five of them had simple lesions of the anterior descending artery. The proportion of patients in the non-surviving group who had a simple anterior descending vessel was higher than that in the surviving group, and there was a statistical difference between the two $(P<0.05)$. (Table 2$)$ Comparison of perioperative data between the two groups: In the survival group, the proportion of patients undergoing coronary artery bypass, complete revascularization and revascularization of culprit vessels was much higher than that in the non-survival group $(P<0.05)$. The emergency operation rate in the survival group was lower than that in the non-survival group $(P$ $<0.05$ ). The survival time and long-term survival time of the patients in the survival group were significantly longer than those in the non-survival group. There was a statistical difference between the two groups $(P<0.05)$, but the incidence of low cardiac output and death in the hospital in the survival group was lower than the non-surviving group. There was a significant statistical difference between the two $(P<$ 0.05 ). (Table 3)Regression analysis of risk factors influencing 30-day mortality: Logistic regression analysis showed that complete revascularization $(\mathrm{OR}=0.021,95 \%$ CI 0.001 $0.374, P=0.009)$ and recanalization of culprit vessels $(\mathrm{OR}=$ $0.045,95 \%$ CI $0.004-0.548, P=0.015$ ) were independent risk factors for 30-day mortality. (Table 4)

The long-term follow up of the patients: Telephone follow up was conducted on surviving patients in the hospital, and the follow-up time was from three months to 136 months. The K-M survival curve showed that during the follow-up period, the long-term survival rate of patients after surgery and complete revascularization was significantly higher than that of patients with conservative drug treatment and incomplete revascularization, and the difference was statistically significant $(P<0.05)$. (Figure 1) (Figure 2)

AMI with VSR is a rare and dangerous complication that often occurs in acute ST segment elevation myocardial infarction [Ying 2017]. VSR mostly occurs within one week after AMI. The patient has a sudden left ventricular shunt at the ventricular level, increased cardiac load, and rapid heart failure, and cardiogenic shock, etc., which in turn causes severe pulmonary congestion and/or hemodynamic deterioration. The effect of conservative drug treatment is extremely poor. At the same time, due to the poor texture of myocardial tissue, the difficulty of suture and the high incidence of low cardiac output after myocardial infarction,

Table 2. Patients' clinical and angiographic data

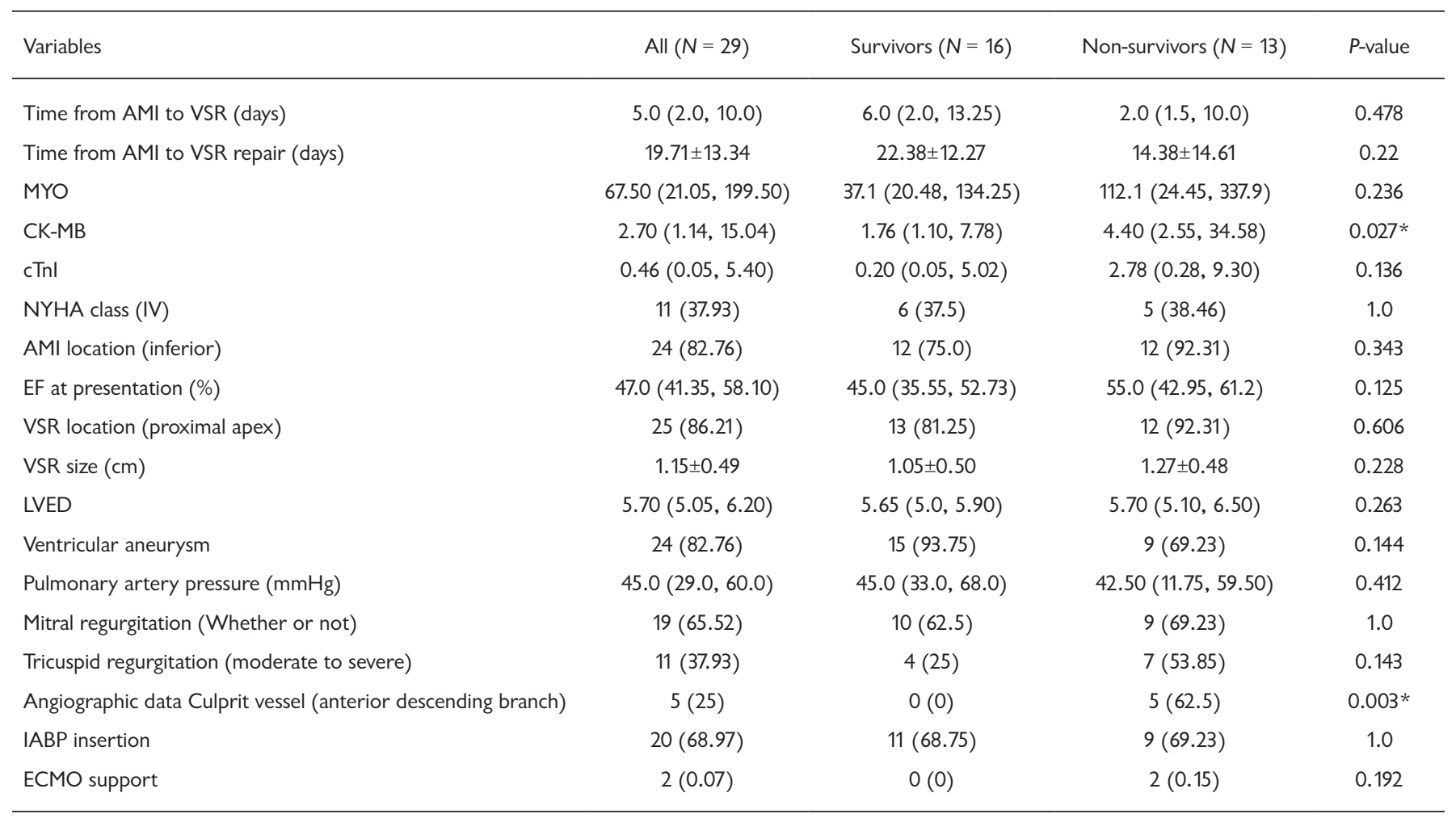

AMI, acute myocardial infarction; VSR, ventricular septal rupture; MYO, Myoglobin; CK-MB, creatine kinase; cTnl, Troponin I; NYHA, New York Heart Association; EF, ejection fraction; LVED, left ventricular end diastolic diameter; IABP, intraaortic balloon counter pulsation; ECMO, extracorporeal membrane oxygenator. *Statistically significant 
Table 3. Perioperative data

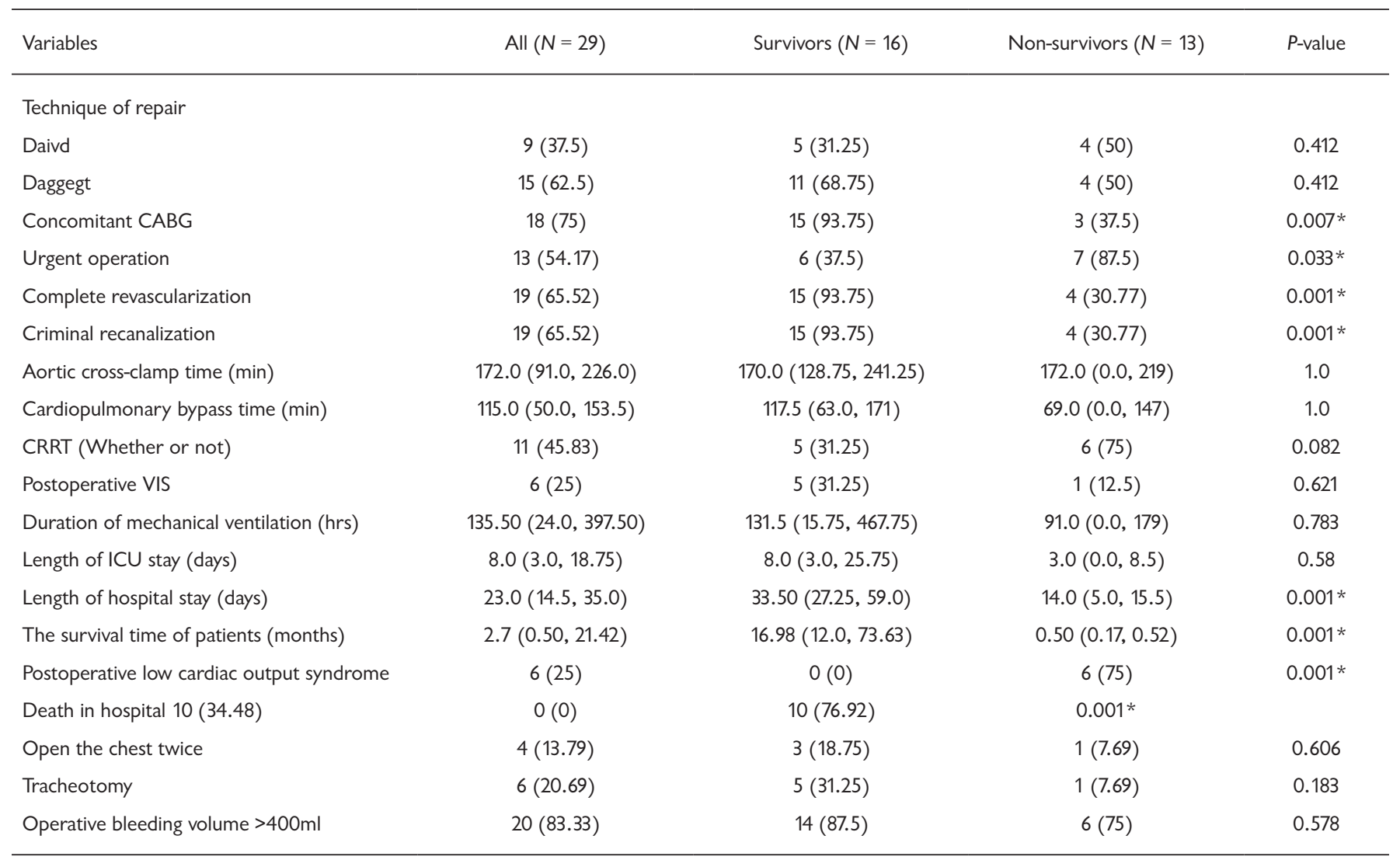

CABG, coronary artery bypass grafting; ICU, intensive care unit; VIS, vasoactive-inotropic score; CRRT, continuous renal replacement therapy. *Statistically significant

Table 4. Associated predictors of 30-day mortality in patients

\begin{tabular}{lccc}
\hline Variables & OR value & $\begin{array}{c}\text { The 95\% confidence } \\
\text { interval }\end{array}$ & $P$-value \\
\hline Gender & .719 & $0.076 \sim 6.793$ & .773 \\
Age & 1.008 & $0.907 \sim 1.120$ & .886 \\
Smoking (Whether or not) & 1.972 & $0.136 \sim 28.501$ & .618 \\
Complete revascularization & 0.021 & $0.001 \sim 0.374$ & $0.009 *$ \\
Criminal recanalization & 0.045 & $0.004 \sim 0.548$ & $0.015^{*}$ \\
AMI location (inferior) & 3.552 & $0.328 \sim 38.495$ & .297 \\
\hline
\end{tabular}

*Statistically significant

surgical operations also face great challenges. In this study, the clinical characteristics and different treatment methods of patients with AMI combined with VSR were compared, the relevant risk factors affecting the 30-day mortality of the patients were analyzed, and the long-term survival rates of the patients were compared.

Previous studies found the risk factors of AMI combined with VSR include old age, female, smoking, combined history of hypertension, anterior wall or large area myocardial infarction, lack of good coronary collateral circulation, etc. [Moreyra 2010; Lopez-Sendon 2010]. In this study, there was no significant difference between the two groups of patients, in terms of age, gender, smoking, history of hypertension, anterior myocardial infarction, and previous myocardial infarction $(P>0.05)$.

Early diagnosis of echocardiography in AMI patients to diagnose and guide clinical treatment is of great value to the prognosis of patients. Echocardiography can be used to determine the location, size, end diastolic diameter of left ventricle, whether there is ventricular aneurysm, pulmonary artery pressure and whether there is bicuspid valve regurgitation, etc., and to preliminarily evaluate the cardiac function and prognosis of patients [Gueret 2008]. In this study, echocardiography showed that most of the perforations were located near the apex of the interventricular septum, but there was no significant difference between the two groups in the location, size, ejection fraction, end diastolic diameter of the left ventricle, whether the VSR was associated with aneurysm, pulmonary artery pressure and whether the bicuspid or tricuspid valve returned $(P>0.05)$.

If the condition of the VSR patient allows, complete coronary angiography before surgery can determine the relevant coronary artery disease, determine whether to perform 


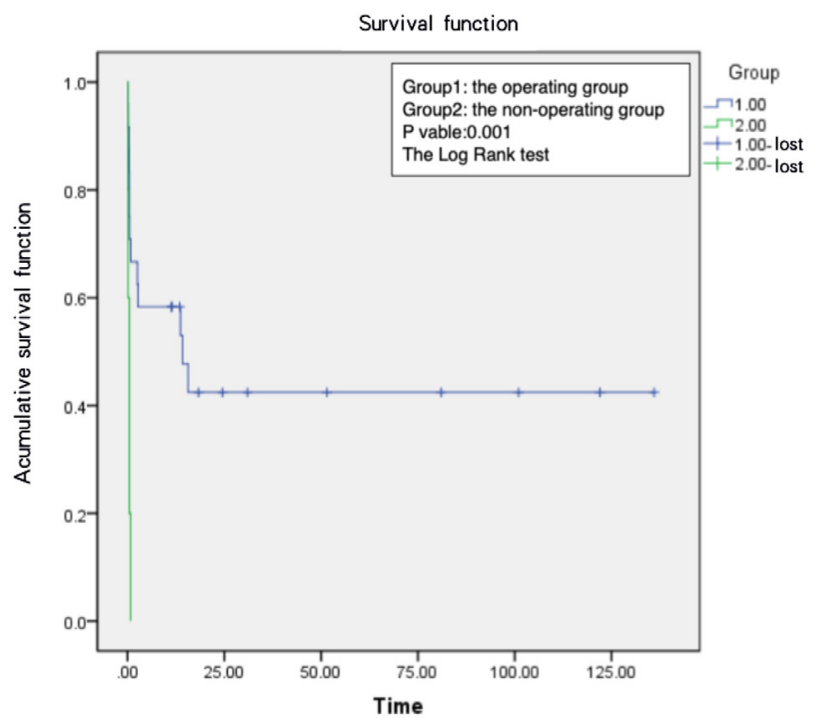

Figure 1. Comparison of long-term survival rate between the operating group and the non-operating group.

coronary artery bypass grafting during the same period, and determine the corresponding target vessel site. Some studies suggest that if the stenosis degree of the main coronary artery and its branches is more than $50 \%$ and the blood supply area is a non-infarcted area, coronary artery bypass grafting should be performed at the same time [Labrousse 2002; Pretre 2000]. Previous studies have found that VSR is mostly a single vessel disease, and the infarct related vessels mostly are anterior descending [Hayashi 2005]. We compared the coronary angiography results of the two groups of patients and found that the proportion of patients in the survival group who underwent coronary angiography before surgery was 75\% (12/16), all of which were coronary vascular lesions of two or more. The ratio of pulse angiography was $61.5 \%$ (8/13), including five cases of anterior descending branch lesions alone. The proportion of patients in the non-surviving group who had a simple anterior descending vessel was higher than that in the surviving group, and there was a statistical difference between the two groups $(P<0.05)$, which is consistent with the results of previous studies, and most of the infarct-related blood vessels are completely occluded, lacking effective collateral circulation, the infarct size is large, the degree of myocardial ischemia in the patient is more serious than that in the survival group, and eventually the 30-day mortality rate of the patient is more obvious than that in the survival group rise. Therefore, patients with VSR should actively improve coronary angiography before surgery to clarify coronary vascular lesions to decide whether to perform coronary artery bypass grafting during the same period.

Surgical operation is an effective method to reduce the hospital mortality of VSR patients, especially for those with a complicated condition and large perforation area, and it is difficult to intervene through intervention means. Due to the high risk of ventricular septal perforation, the timing of surgery is particularly important. However, determining the best

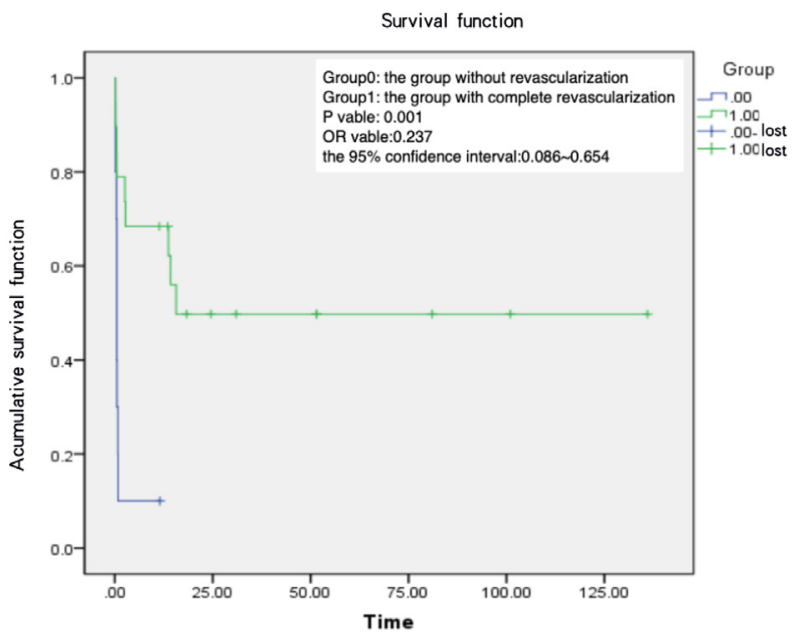

Figure 2. Comparison of long-term survival rate between patients with complete revascularization and those without revascularization.

operation time for VSR always has been controversial. Previous studies have suggested that the shorter the time from diagnosis to operation, the higher the operative mortality rate [Noguchi 2012]. Papalexopoulou and other researchers believe that under the condition of stable hemodynamics, surgery should be delayed as much as possible [Papalexopoulou 2013]; however, current European guidelines recommend that patients with VSR should undergo surgery as soon as possible to reduce the incidence of heart failure [O'Gara 2013; Ryan 1999]. In the early period of perforation, the edges of the perforation were unclear, and the surrounding tissues were brittle, which increased the difficulty of surgical repair and the risk of repair failure [Muehrcke 1992]; the purpose of delayed surgery was to reduce the difficulty of repair. It may cause some patients to die due to the worsening of circulatory conditions, while waiting for surgery, and lose the chance of surgery. Maoutakis et al.'s large sample study found that the mortality of surgery within one week of AMI combined with VSR was $54.1 \%$, while the mortality of AMI combined with VSR for more than one week before surgery was only $18.4 \%$ [Maoutakis 2012]. We compared the perioperative data of the two groups and found that the emergency operation rate of the survival group was lower than that of the non-survival group. There was a statistical difference between the two groups $(\mathrm{P}<0.05)$, from the diagnosis of VSR to the operation time. The average time of the survival group was 22.38 \pm 12.27 days, and the average time of the non-survival group was $14.38 \pm 14.61$ days, although there was no significant statistical difference between the two groups $(P>0.05)$. However, the time from the diagnosis of the VSR to the operation in the survival group was significantly longer than that in the non-survival group, and the emergency surgery rate of the survival group was significantly lower than that of the nonsurvival group. The above two points further confirmed the importance of the timing of VSR surgery.

Previous studies have shown that the rapid deterioration of preoperative circulatory state is an important risk factor for 
poor perioperative prognosis of patients [Cerin 2003], while critical preoperative state is closely related to the time of perforation after myocardial infarction, the degree of interventricular septum involvement and perforation [Menon 2000]. In general, VSR usually occurs in the first week after AMI, with an average time of 3-5 days [Poui 2008]. This study shows that the overall average time of perforation is five days $(2.0,10.0)$. Compared with the time from AMI to VSR diagnosis in the two groups, the average time of survival group is six days $(2.0,13.25)$, while the average time of non-survival group is two days $(1.5,10.0)$. Although there was no significant statistical difference between the two groups $(P>0.05)$, it also supported the previous point to some extent.

Previous studies have found that complete revascularization and revascularization of culprit vessels in VSR patients can reduce 30-day mortality and improve long-term prognosis [Barker 2003; Pretre 2000; Cox 1996]. Lundblad et al. also found that simultaneous bypass during operation can reduce the early and long-term mortality of VSR patients [Lundblad 2009]. Perotta et al. also reported that simultaneous bypass can reduce the mortality of VSR patients [Perotta 2009]. In this study, we found that the proportion of patients in the survival group who underwent coronary artery bypass, complete revascularization and culprit revascularization at the same time was much higher than that in the non-survival group, and there was a significant statistical difference between the two groups $(P<0.05)$. This was consistent with the results reported in the previous studies, and fully reflected the importance of the simultaneous revascularization and culprit revascularization in patients with AMI and VSR during the operation. Logistic regression analysis showed that complete revascularization $(\mathrm{OR}=0.021,95 \%$ confidence interval $0.001-0.374, P=0.009)$ and recanalization of culprit vessels $(\mathrm{OR}=0.045,95 \%$ confidence interval $0.004-0.548, P=$ $0.015)$ were independent risk factors for 30-day mortality of VSR patients. Telephone follow up of surviving patients in the hospital was conducted between three months and 136 months. The KM survival curve shows that during the follow-up period, the long-term survival rate of patients after surgical operation and complete revascularization is significantly higher than that of conservative medical treatment and patients who have not undergone complete revascularization despite surgical treatment. The results of this study further confirm the previous research. The reason may be that complete revascularization and revascularization of culprit vessels can effectively improve the blood supply of myocardial tissue, thus reducing the 30 -day mortality rate of VSR patients and improving the long-term prognosis, but there is no retrospective study to confirm the direct causal relationship between them. If we want to confirm this, we need to conduct a largescale prospective control trial, and the patients in the control group must deliberately ignore the same period of coronary artery bypass grafting, it is obvious that this prospective control trial is never possible [Runar 2009]. From the K-M survival curve, it can be seen that the long-term prognosis of VSR patients treated by surgery and complete revascularization is better than that of patients treated by drug conservative therapy and surgery but not complete revascularization
$(P<0.05)$. It is also confirmed once again that drug treatment can only be used as a transitional treatment for the VSR patients, but not as an independent treatment. Surgical surgery is still the preferred treatment option for patients with AMI and VSR.

In summary, our study found that whether complete revascularization and revascularization of criminals are independent risk factors that affect the 30-day mortality rate in patients with AMI and VSR. Surgery and complete revascularization can significantly improve the hospital survival rate and the long-term prognosis of the VSR patients. Since this study is a single-center retrospective study, it is inevitable that there is a certain bias. The sample size is small, and the statistical power also will be affected to a certain extent. In the future, a larger sample size and longer follow-up time for multiple centers will be needed to further validate our findings.

\section{ACKNOWLEDGEMENT}

The fund: Strategy of computer assisted individualized coronary artery bypass grafting (RDY2019-32).

\section{REFERENCES}

Barker TA, Ramnarine IR, Woo EB, Grayson AD, Au J, Fabri BM, et al. 2003. Repair of post-infarct ventricular septal defect with or without coronary artery bypass grafting in the northwest of England: a 5-year multi-institutional experience. Eur J Cardiothorac Surg. 24:940-6.

Cerin G. 2003. Surgical treatment of ventricular septal defect complicating acute myocardial infarction. Experience of a north Italian referral hospital. Cardiovasc Surg. 11(2):149-154.

Coskun KO, Coskun ST, Popov AF, et al. 2009. Experiences with surgical treatment of ventricle septal defect as a post infarction complication. J Cardiothorac Surg. 4:3.

Cox FF, Plokker HW, Morshuis WJ, Kelder JC, Vermeulen FE. 1996. Importance of coronary revascularization for late survival after postinfarction ventricular septal rupture. A reason to perform coronary angiography prior to surgery. Eur Heart J. 17:1841-5.

Crenshaw BS, Granger CB, Birnbaum Y, Pieper KS, Morris DC, Kleiman NS, et al. 2000. Risk factors, angiographic patterns, and outcomes in patients with ventricular septal defect complicating acute myocardial infarction. Circulation. 101:27-32.

Gueret P, Khalife K, Jobic Y, et a1. 2008. Echocardiographic assessment of the incidence of mechanical complications during the early phase of myocardial infarction in the reperfusion era: a French muhieentre prospective registry [J]. Archives of Cardiovascular Diseases. 101 (1): 41—47.

Hayashi T, Hirano Y, Takai H, et a1. 2005. Usefulness of ST— segment elevation in the inferior leads in predicting ventricular septal rupture in patients with anterior wall acute myocardial infarction[J]. Am J Cardiol. 96(8): 1037-1041.

Labrousse L, Choukroun E, Chevalier JM, et a1. 2002. Surgery for postinfarction ventricular septal defect (VSD): risk factors for hospital death and long term results [J]. Eur J CardiothoracSurg. 21(4): 725-732.

Lopez-Sendon J, Gurfinkel EP, Lopez de Sa E, et al. 2010. Factors related to heart rupture in acute coronary syndromes in the Global Registry of 
Acute Coronary Events [J]. Eur Heart J. 31(12):1449-1456.

Lundblad R, Abdelnoor M, Geiran OR, Svennevig JL. 2009. Surgical repair of postinfarction ventricular septal rupture: risk factors of early and late death. J Thorac Cardiovasc Surg. 137(4):862-8.

Maoutakis GJ, Zha0 Y, Geoe TJ, et a1. 2012. Suicalrepair of ventricular septal debctaher myocardial infarction: outcomes from the society of thoracic surgeons national database[J]. Ann Thomcsu. 94(2):436—444.

Menon V, Webb JG, Hillis LD, et al. 2000. Outcome and profile of ventricular septal rupture with cardiogenic shock after myocardial infarction: A report from the SHOCK Trial Registry. J Am CollCardiol. 36(3 SUPPL. A):1110-1116.

Moreyra AE, Huang MS, Wilson AC, et al. 2010. Trends in incidence and mortality rates of ventricular septal rupture during acute myocardial infarction. Am J Cardiol. 106 (8) 1095-1100.

Muehrcke DD, Daggett WM Jr, Buckley MJ, Akins CW, Hilgenberg AD, Austen WG. 1992. Postinfarct ventricular septal defect repair: effect of coronary artery bypass grafting. Ann Thorac Surg. 54:876-82.

Noguchi K, Yamaguchi A, Naito K, Yuri K, Adachi H. 2012. Short-term and long-term outcomes of postinfarction ventricular septal perforation. Gen Thorac Cardiovasc Surg. 60:261-7.

O'Gara PT, Kushner FG, Ascheim DD, Casey Jr DE, Chung MK, De Lemos JA, et al. 2013. 2013 ACCF/AHA Guideline for the management of ST-elevation myocardial infarction. Circulation. 127:e362-425.

Pang PY, Sin YK, Lim CH, et al. 2013. Outcome and survival analysis of surgical repair of post-infarction ventricular septal rupture. J Cardiothorac Surg. 8:44.

Papalexopoulou N, Young CP, Attia RQ. 2013. What is the best timing of surgery in patients with post-infarct ventricular septal rupture? Interact Cardiovasc Thorac Surg. 16(2):193-196.

Perotta S, Lentini S. 2009. In patients undergoing surgical repair of postinfarction ventricular septal defect, does concomitant revascularization improve prognosis? Interact Cardiovasc Thorac Surg. 9(5):879-87.

Poui_Sen SH, Praestholm M, Munk K, et a1. 2008. Ventricular septal rupture complicating acute myocardial infarction: clinical characteristics and contemporary outcome[J]. Ann ThoraeSurg. 85(5): 1591-1596.

Pretre R, Ye Q, Grinenfelder J, et al. 2000. Role of myocardial revascularization in postinfarction ventricular septal rupture[J]. Ann Thorac Surg. 69 (1): $51-55$.

Runar Lundblad, MD, PhDa, Michel Abdelnoor, PhDb. 2009. Surgical repair of postinfarction ventricular septal rupture: Risk factors of early and late death. The Journal of Thoracic and Cardiovascular Surgery April.

Ryan TJ, Antman EM, Brooks NH, Califf RM, Hillis LD, Hiratzka LF, et al. 1999. 1999 update: ACC/AHA guidelines for the management of patients with acute myocardial infarction. J Am Coll Cardiol. 34:890-911.

Ying S, Yin L. 2017. Characteristics and treatment progress of ventricular septal perforation after acute myocardial infarction. Tianjin Pharmaceuticals, 45 (11): 1149-1152. 